\begin{tabular}{|c|l|}
\hline Title & Scaling limit for a generalization of the Nelson model and its application to nuclear physics \\
\hline Author(s) & Suzuki, A kito \\
\hline Citation & Hokkaido University Preprint Series in Mathematics, 782, 1-24 \\
\hline Issue Date & 2006-04 10 \\
\hline DOI & 10.14943/83932 \\
\hline Doc URL & http:/hdl.handle.net/2115/69590 \\
\hline Type & bulletin (article) \\
\hline File Information & pre782.pdf \\
\hline
\end{tabular}

Instructions for use 


\title{
Scaling limit for a generalization of the Nelson model and its application to nuclear physics
}

\author{
Akito Suzuki \\ Department of Mathematics, \\ Hokkaido University, \\ Sapporo 060-0810, Japan \\ e-mail: akito@math.sci.hokudai.ac.jp
}

April 10, 2006

\begin{abstract}
We study a mathematically rigorous derivation of a quantum mechanical Hamiltonian in a general framework. We derive such a Hamiltonian by taking a scaling limit for a generalization of the Nelson model, which is an abstract interaction model between particles and a Bose field with some internal degrees of freedom.

Applying it to a model for the field of the nuclear force with an isospin, we obtain a Schrödinger Hamiltonian with a matrix valued potential, describing an effective interaction between nucleons.
\end{abstract}

Keywords: scaling limit, weak coupling limit, GSB model, Nelson model, effective potential, isospin, nuclear force

AMS Subject Classification: $81 \mathrm{Q} 10$

\section{Introduction}

We consider a scaling limit of an abstract quantum field theoretical Hamiltonian of particles coupled to a Bose field with some internal degrees of freedom such as a spin or an isospin. The purpose of this paper is to derive a quantum mechanical Hamiltonian in the scaling limit of such a quantum field theoretical Hamiltonian in a general framework. 
A typical example of scaling limits is the weak coupling limit for a model of norelativistic quantum particles coupled to a Bose field whose Hamiltonian is given by

$$
H=-\frac{1}{2 M} \Delta \otimes I+I \otimes H_{\mathrm{b}}+g H_{\mathrm{I}},
$$

where $M>0$ denotes the mass of the particles, $\Delta$ the generalized Laplacian, $H_{\mathrm{b}}$ the free Hamiltonian of the Bose field, $H_{\mathrm{I}}$ an interaction between the particles and the Bose field, $g \in \mathbb{R}$ a coupling constant which represents the strength of the interaction. A scaled Hamiltonian of $H$ is introduced by

$$
H(\Lambda)=-\frac{1}{2 M} \Delta \otimes I+\Lambda^{2} I \otimes H_{\mathrm{b}}+g \Lambda H_{\mathrm{I}}, \Lambda>0 .
$$

One can show that, under suitable conditions, there exists a symmetric operator $V_{\text {eff }}$, called an effective potential, such that

$$
\text { S- } \lim _{\Lambda \rightarrow \infty}(H(\Lambda)-z)^{-1}=\left(-\frac{1}{2 M} \Delta+V_{\text {eff }}-z\right)^{-1} \otimes P_{0}
$$

for all $z \in \mathbb{C} \backslash \mathbb{R}$, where $P_{0}$ denotes the orthogonal projection onto ker $H_{\mathrm{b}}$. Physically, a vector belonging to the subspace ker $H_{\mathrm{b}}$ represents the vacuum of the free Bose field. Therefore one obtains a quantum mechanical Hamiltonian, called a Schrödinger Hamiltonian, in the vacuum of the free Bose field in the resolvent sense. According to Davies [5], the limit (1.1) is the weak coupling limit at the same time as the mass of the particles becomes infinity, since we can write

$$
H(\Lambda)=\Lambda^{2}\left(-\frac{1}{2 M \Lambda^{2}} \Delta \otimes I+I \otimes H_{\mathrm{b}}+\frac{g}{\Lambda} H_{\mathrm{I}}\right)
$$

where the factor $\Lambda^{2}$ on the whole Hamiltonian is interpreted as a time scaling. However, Davies dealt with spinless particles and bosons. Hiroshima [6, 7] investigated the weak coupling limit and removing ultraviolet cutoff for the Nelson model, which describes spinless particles interacting with spinless bosons.

On the other hand, Arai [1] studied scaling limits for a spin-boson interaction model, called the spin boson model, and a model in nonrelativistic quantum electrodynamics, called the Pauli-Fierz model, in the dipole approximation without the self-interaction of photons. The methods in [1] have been extended to the generalized spin boson (GSB) model [3] and the Pauli-Fierz model with the self-interaction of photons ([8] and the refernces therein).

In this paper, we study a scaling limit for a generalization of the Nelson model, which describes particles coupled to a Bose field with some internal 
degrees of freedom. Various branches of physics, such as nuclear physics and condensed matter physics, have many examples of the model, and the interaction $H_{\mathrm{I}}$ depends on models $[3,13]$. From this point of view, it seems natural to consider scaling limits of these general models under conditions as weak as possible. As a result, we are now able to derive an effective potential that is an operator-valued potential in the weak coupling limit. Note that, since the Nelson model has no internal degree of freedom, the effective potential is a scalar potential. However, in nuclear physics, matrix valued potentials appear as effective potentials. A new feature of our work is in that a quantum mechanical Hamiltonian with such a potential is derived.

This paper is organized as follows. Sec. 2 is devoted to a proof of an abstract scaling limit theorem. We introduce some notations, and modify a scaling limit theorem established in $[1,6,13]$ for later use. In Sec. 3, we define our model and state the main theorem of this paper. We prove the main results in Sec. 4. In the last section, we discuss an application to a model in nuclear physics.

\section{Preliminaries}

In this section, we describe an abstract scaling limit theorem $([1,6,13])$ in a convenient form to establish the scaling limit for our model. We denote the inner product and the associated norm of a Hilbert space $\mathcal{L}$ by $\langle\cdot, \cdot\rangle_{\mathcal{L}}$ and $\|\cdot\|_{\mathcal{L}}$, respectively. If there is no danger of confusion, we omit the subscript $\mathcal{L}$ in $\langle\cdot, \cdot\rangle_{\mathcal{L}}$ and $\|\cdot\|_{\mathcal{L}}$. Moreover, the domain and range of an operator $T$ is denoted by $D(T)$ and $\operatorname{Ran}(T)$, respectively.

\section{$2.1 \quad$ Uniform relative boundedness}

To begin with, we introduce the following notions which are useful for describing a condition of a scaling limit theorem.

Definition 2.1 Let $\mathcal{L}$ be a Hilbert space, $t_{0}$ a point in an interval $I_{0} \subset$ $[-\infty,+\infty]$, and $L(t)$ and $M(t)\left(t \in I_{0}\right)$ operators on $\mathcal{L}$ satisfying

$$
\bigcap_{t \in I_{0}} D(L(t)) \neq \emptyset \text {. }
$$

(1) We say that $M(t)$ is $L(t)$-bounded uniformly near $t_{0}$ if there exist a neighborhood $I \subset I_{0}$ of $t_{0}$ and constants $a, b \geq 0$ such that for any $t \in I \backslash\left\{t_{0}\right\}$, $D(M(t)) \supset D(L(t))$ and

$$
\|M(t) \Psi\| \leq a\|L(t) \Psi\|+b\|\Psi\|, \quad \Psi \in D(L(t)) .
$$


(2) We say that $M(t)$ is $L(t)$-infinitesimally small uniformly near $t_{0}$ if for any $\varepsilon>0$, there exist an interval $I(\varepsilon) \subset I_{0}$ and a constant $b(\varepsilon)$ such that for any $t \in I(\varepsilon) \backslash\left\{t_{0}\right\}, D(M(t)) \supset D(L(t))$ and

$$
\|M(t) \Psi\| \leq \varepsilon\|L(t) \Psi\|+b(\varepsilon)\|\Psi\|, \quad \Psi \in D(L(t)) .
$$

Note that, from the Kato-Rellich Theorem, if $M(t)$ is $L(t)$-infinitesimally small uniformly near $t_{0}$, then $L(t)+M(t)$ is self-adjoint on $D(L(t))$ for all $t \in I \backslash\left\{t_{0}\right\}$ with some neighborhood $I$ of $t_{0}$, and moreover, if $L(t)$ is bounded from below, then so is $L(t)+M(t)$.

\subsection{Abstract scaling limit}

Let $A$ be a non-negative self-adjoint operator on a Hilbert space $\mathcal{H}$ and $B$ a non-negative self-adjoint operator on a Hilbert space $\mathcal{K}$ with

$$
\text { ker } B \neq\{0\} .
$$

We denote by $P_{\mathrm{B}}$ the orthogonal projection onto $\operatorname{ker} B$ from $\mathcal{K}$. Let $\left\{C_{\Lambda}\right\}_{\Lambda>0}$ be symmetric operators on $\mathcal{X}:=\mathcal{H} \otimes \mathcal{K}$. Put

$$
K(\Lambda):=K_{0}(\Lambda)+C_{\Lambda}
$$

where

$$
K_{0}(\Lambda):=A \otimes I+\Lambda I \otimes B
$$

We consider the scaling limit of $K(\Lambda)$.

Put

$$
K_{0}:=A \otimes I+I \otimes B .
$$

The following lemma on unifom relative boundedness is useful for proving scaling limits.

Lemma 2.1 If $C_{\Lambda}$ is $I \otimes B$-bounded uniformly near $\infty$, then $C_{\Lambda}$ is $K_{0}(\Lambda)$ infinitesimally small uniformly near $\infty$. Also, if $C_{\Lambda}$ is $K_{0}$-infinitesimally small uniformly near $\infty$, then $C_{\Lambda}$ is $K_{0}(\Lambda)$-infinitesimally small uniformly near $\infty$.

Note that the followimg lemma holds.

Lemma 2.2 Let $C_{\Lambda}$ be $K_{0}(\Lambda)$-infinitesimally small uniformly near $\infty$. Then for $z \in \mathbb{C} \backslash[0, \infty)$

$$
\text { S- } \lim _{\Lambda \rightarrow \infty} C_{\Lambda}\left(K_{0}(\Lambda)-z\right)^{-1} I \otimes\left(I-P_{\mathrm{B}}\right)=0 .
$$


Proof. It is easy to see that for any $\varepsilon>0$ there exists a constant $\Lambda_{0}(\varepsilon)$ such that for all $\Lambda>\Lambda_{0}(\varepsilon)$

$$
\begin{aligned}
& \left\|C_{\Lambda}\left(K_{0}(\Lambda)-z\right)^{-1} I \otimes\left(I-P_{\mathrm{B}}\right) \Psi\right\| \\
& \quad \leq \varepsilon\left\|I \otimes\left(I-P_{\mathrm{B}}\right) \Psi\right\|+(\varepsilon|z|+b(\varepsilon))\left\|\left(K_{0}(\Lambda)-z\right)^{-1} I \otimes\left(I-P_{\mathrm{B}}\right) \Psi\right\|, \quad \Psi \in \mathcal{X} .
\end{aligned}
$$

Since the second term on the right hand side converges to zero as $\Lambda \rightarrow \infty$, we obtain the desired result.

Now, we can prove the following scaling limit theorem.

Theorem 2.3 (scaling limit $[1,6,13]$ ) Suppose that $C_{\Lambda}$ is $K_{0}(\Lambda)$-infinitesimally small uniformly near $\infty$ and there exists a symmetric operator $C$ on $\mathcal{X}$ such that $D(C) \supset D(A) \otimes \operatorname{ker} B$ and for all $z \in \mathbb{C} \backslash \mathbb{R}$

$$
\text { S- } \lim _{\Lambda \rightarrow \infty} C_{\Lambda}(A-z)^{-1} \otimes P_{\mathrm{B}}=C(A-z)^{-1} \otimes P_{\mathrm{B}} .
$$

Then, the following (1)-(3) hold.

(1)For any $\Lambda>\Lambda_{0}$ with some $\Lambda_{0}, K(\Lambda)$ is self-adjoint on $D\left(K_{0}\right)$ and bounded from below uniformly in $\Lambda>\Lambda_{0}$. Moreover, it is essentially self-adjoint on any core for $K_{0}$.

(2)The operator

$$
K_{\mathrm{eff}}:=A \otimes I+\left(I \otimes P_{\mathrm{B}}\right) C\left(I \otimes P_{\mathrm{B}}\right)
$$

is self-adjoint on $D(A \otimes I)$ and bounded from below. Moreover, it is essentially self-adjoint on any core for $A \otimes I$.

(3)For any $z \in \mathbb{C} \backslash \mathbb{R}$ or $z<0$ with $|z|$ sufficiently large

$$
\text { S- } \lim _{\Lambda \rightarrow \infty}(K(\Lambda)-z)^{-1}=\left(K_{\mathrm{eff}}-z\right)^{-1} I \otimes P_{\mathrm{B}}
$$

Proof. It is easy to prove (1) and (2). (See [13].) By Lemma 2.2 and (2.2), one can easily show that

$$
\text { S- } \lim _{\Lambda \rightarrow \infty} C_{\Lambda}\left(K_{0}(\Lambda)-z\right)^{-1}=C(A-z)^{-1} \otimes P_{\mathrm{B}}
$$

There exists a constant $\epsilon_{0}<0$ such that $K_{\Lambda} \geq \epsilon_{0}$, for all $\Lambda>\Lambda_{0}$. Let $|z|>0$ be sufficiently large so that $z<\epsilon_{0}<0$. Iterating the second resolvent formula, we have

$$
(K(\Lambda)-z)^{-1}=\sum_{n=0}^{N}(-1)^{n}\left(K_{0}(\Lambda)-z\right)^{-1} T_{\Lambda}{ }^{n}+R_{N}(\Lambda),
$$


where

$$
T_{\Lambda}:=C_{\Lambda}\left(K_{0}(\Lambda)-z\right)^{-1}
$$

and

$$
R_{N}(\Lambda):=(-1)^{N+1}\left(K_{\Lambda}-z\right)^{-1} T_{\Lambda}^{N+1}
$$

Since $C_{\Lambda}$ is $K_{0}(\Lambda)$-infinitesimally small uniformly near $\infty$,

$$
\left\|T_{\Lambda}\right\| \leq 2 \varepsilon+\frac{b(\varepsilon)}{|z|}
$$

for all $\Lambda>\Lambda_{0}$ with some $\Lambda_{0}$. Hence, taking $\varepsilon<1 / 2$,

$$
\left\|T_{\Lambda}\right\|<1
$$

uniformly in $\Lambda>\Lambda_{0}$, if $|z|$ is surfficiently large. Since one can easily prove

$$
\left\|R_{N}(\Lambda)\right\| \leq \frac{\left\|T_{\Lambda}\right\|^{N+1}}{\left|\epsilon_{0}-z\right|}
$$

it follows that

$$
(K(\Lambda)-z)^{-1}=\sum_{n=0}^{\infty}(-1)^{n}\left(K_{0}(\Lambda)-z\right)^{-1} T_{\Lambda}{ }^{n}
$$

in operator norm uniformly in $\Lambda>\Lambda_{0}$. Taking $\Lambda \rightarrow \infty$ and using (2.4), we get the desired result with $z<0,|z|$ being sufficiently large. (For the details, see $[1$, Theorem 2.2].) The proof in the case where $z$ is not real is similar to that in [1].

We denote by $\otimes_{\text {alg }}$ the algebraic tensor product. We can state the scaling limit theorem under easier conditions:

Corollary 2.4 Suppose that $C_{\Lambda}$ is $K_{0}(\Lambda)$-infinitesimally small uniformly near $\infty$ and there exists a closed symmetric operator $C$ on $\mathcal{X}$ satisfying the following condition:

There exists a core $\mathcal{D}$ for $A \otimes I$ such that $D(C) \supset \mathcal{D} \otimes_{\text {alg }} \operatorname{ker} B$ and for all $\Psi \in \mathcal{D} \otimes_{\text {alg }} \operatorname{ker} B$

$$
\text { S- } \lim _{\Lambda \rightarrow \infty} C_{\Lambda} \Psi=C \Psi
$$

Then, (1)-(3) in Theorem 2.3 hold.

Proof. We need only to prove (2.2). It is easy to see that for any $\Lambda \geq \Lambda_{0}$ with some $\Lambda_{0}$

$$
\left\|C_{\Lambda}(A-z)^{-1} \otimes P_{\mathrm{B}} \Psi\right\| \leq\left[\varepsilon+(\varepsilon|z|+b(\varepsilon))\left\|(A-z)^{-1}\right\|\right]\|\Psi\|, \quad \Psi \in \mathcal{X} .
$$


Hence, $C_{\Lambda}(A-z)^{-1} \otimes P_{\mathrm{B}}$ is a bounded operator with

$$
\sup _{\Lambda \geq \Lambda_{0}}\left\|C_{\Lambda}(A-z)^{-1} \otimes P_{\mathrm{B}}\right\|<\infty .
$$

Put

By the injectivity of $(A-z)^{-1}$,

$$
\mathcal{L}:=(A-z) \mathcal{D} .
$$

$$
(A-z) \mathcal{D}=\left\{u \in \mathcal{H} \mid(A-z)^{-1} u \in \mathcal{D}\right\} .
$$

Since $\mathcal{D}$ is core for $A$, for every $\Psi \in D(A)$ there exists a sequence $\left\{\Psi_{n}\right\} \subset \mathcal{D}$ such that

$$
\Psi_{n} \longrightarrow \Psi, \quad A \Psi_{n} \longrightarrow A \Psi
$$

Thus we obtain that

$$
\overline{(A-z) \mathcal{D}}=\operatorname{Ran}(A-z)=\mathcal{H} .
$$

Hence, $\mathcal{L}$ is dense in $\mathcal{H}$. For all $\Psi \in \mathcal{L} \otimes_{\text {alg }} \mathcal{K}$,

$$
(A-z)^{-1} \otimes P_{\mathrm{B}} \Psi \in \mathcal{D} \otimes_{\text {alg }} \operatorname{ker} B
$$

and

$$
\text { S- } \lim _{\Lambda \rightarrow \infty} C_{\Lambda}(A-z)^{-1} \otimes P_{\mathrm{B}} \Psi=C(A-z)^{-1} \otimes P_{\mathrm{B}} \Psi .
$$

Therefore, taking $\Lambda \rightarrow \infty$ in (2.9),

$\left\|C(A-z)^{-1} \otimes P_{\mathrm{B}} \Psi\right\| \leq\left[\varepsilon+(\varepsilon|z|+b(\varepsilon))\left\|(A-z)^{-1}\right\|\right]\|\Psi\|, \quad \Psi \in \mathcal{L} \otimes_{\mathrm{alg}} \mathcal{K}$.

By closedness of $C, C(A-z)^{-1} \otimes P_{\mathrm{B}}$ is a closed operator. Since $\mathcal{L} \otimes_{\text {alg }} \mathcal{K}$ is dense in $\mathcal{X}, C(A-z)^{-1} \otimes P_{\mathrm{B}}$ is bounded on $\mathcal{H}$. By (2.10) and the density of $\mathcal{L} \otimes_{\text {alg }} \mathcal{K}$ again, we obtain $(2.2)$.

\section{Definitions and the main theorem}

\subsection{Boson Fock space}

To describe a Bose field, one uses the Boson Fock space over a complex Hilbert space $\mathcal{K}$ :

$$
\begin{aligned}
\mathcal{F}_{\mathrm{b}}(\mathcal{K}): & =\bigoplus_{n=0}^{\infty} \bigotimes_{\mathrm{s}}^{n} \mathcal{K} \\
& =\left\{\psi=\left\{\Psi^{(n)}\right\}_{n=0}^{\infty} \mid n \geq 0, \quad \Psi^{(n)} \in \bigotimes_{\mathrm{s}}^{n} \mathcal{K}, \quad \sum_{n=0}^{\infty}\left\|\psi^{(n)}\right\|^{2}<\infty\right\},
\end{aligned}
$$


where $\bigotimes_{\mathrm{s}}^{n} \mathcal{K}$ denotes the $n$-fold symmetric tensor product of $\mathcal{K}$ with $\bigotimes_{\mathrm{s}}^{0} \mathcal{K}:=$ $\mathbb{C}$.

The annihilation operator $a(f)(f \in \mathcal{K})$ is a densely defined closed linear operator on $\mathcal{F}_{\mathrm{b}}(\mathcal{K})$ such that, for all $\psi=\left\{\Psi^{(n)}\right\}_{n=0}^{\infty} \in D\left(a(f)^{*}\right),\left(a(f)^{*} \psi\right)^{(0)}=$ 0 and

$$
\left(a(f)^{*} \psi\right)^{(n)}=\sqrt{n} S_{n}\left(f \otimes \Psi^{(n-1)}\right), \quad n \geq 1,
$$

where $S_{n}$ is the symmetrization operator on $\bigotimes^{n} \mathcal{K}\left(S_{n}{ }^{*}=S_{n}, S_{n}{ }^{2}=S_{n}\right.$, $\left.\bigotimes_{s}^{n} \mathcal{K}=S_{n}\left(\bigotimes^{n} \mathcal{K}\right)\right)$. The adjoint $a(f)^{*}$, called the creation operator, and the annihilation operator $a(g)(g \in \mathcal{K})$ obey the canonical commutation relations

$$
\left[a(f), a(g)^{*}\right]=\langle f, g\rangle, \quad[a(f), a(g)]=0, \quad\left[a(f)^{*}, a(g)^{*}\right]=0
$$

for all $f, g \in \mathcal{K}$ on the dense subspace

$$
\begin{array}{r}
\mathcal{F}_{0}(\mathcal{K}):=\left\{\psi \in \mathcal{F}_{\mathrm{b}}(\mathcal{K}) \mid \text { there exists a number } n_{0}\right. \text { such that } \\
\left.\psi^{(n)}=0 \text { for all } n \geq n_{0}\right\},
\end{array}
$$

where $[X, Y]:=X Y-Y X$.

The Segal field operator $\phi(f)(f \in \mathcal{K})$ is defined by

$$
\phi(f):=\frac{a(f)+a(f)^{*}}{\sqrt{2}} .
$$

It is shown that $\phi(f)$ is essentially self-adjoint on $\mathcal{F}_{0}(\mathcal{K})[11, \S \mathrm{X} .7]$. We denote its closure by the same symbol $\phi(f)$.

It follows from (3.1) that, for all $f, g \in \mathcal{K}$,

$$
[\phi(f), \phi(g)]=i \Im\langle f, g\rangle
$$

on $\mathcal{F}_{0}(\mathcal{K})$. Moreover, we have

$$
e^{i \phi(f)} e^{i \phi(g)}=e^{-i \Im\langle f, g\rangle} e^{i \phi(g)} e^{i \phi(f)}, \quad f, g \in \mathcal{K},
$$

which are called the Weyl relations of $\{\phi(f) \mid f \in \mathcal{K}\}[11, \S \mathrm{X} .7]$.

For every self-adjoint operator $T$ on $\mathcal{K}$, one can define a self-adjoint operator $d \Gamma(T)$, called the second quantization of $T$ [10, p.302], by

$$
d \Gamma(T):=\bigoplus_{n=0}^{\infty} T^{(n)},
$$

with $T^{(0)}=0$ and $T^{(n)}$ is the closure of

$$
\left(\sum_{j=1}^{n} I \otimes \cdots \otimes \stackrel{\text { th }}{T} \otimes \cdots \otimes I\right) \mid \bigotimes_{\text {alg }}^{n} D(T) .
$$

If $T$ is non-negative, then so is $d \Gamma(T)$. 


\subsection{Definition of a generalization of the Nelson model}

We consider a model of a quantum system $\mathcal{S}$ coupled to a Bose field with some internal degrees of freedom. The Hilbert space of the system $\mathcal{S}$ is taken to be $L^{2}\left(\mathbb{R}^{d} ; \mathcal{H}\right)$. Here $d \in \mathbb{N}$ and $\mathcal{H}$ is taken to be an arbitary separable complex Hilbert space. In concrete realizations, $\mathcal{S}$ may be a system of quantum particles with some internal degrees of freedom such as spin and isospin. The Hilbert space of the coupled system of $\mathcal{S}$ and the Bose field is given by the tensor product

$$
\mathcal{F}:=L^{2}\left(\mathbb{R}^{d} ; \mathcal{H}\right) \otimes \mathcal{F}_{\mathrm{b}}(\mathcal{K}) \simeq \mathcal{H} \otimes L^{2}\left(\mathbb{R}^{d} ; \mathcal{F}_{\mathrm{b}}(\mathcal{K})\right) .
$$

We assume that $T$ is a non-negative, injective and self-adjoint operator on $\mathcal{K}$. Then, the free Hamiltonian of the Bose field is defined by

$$
H_{\mathrm{b}}:=d \Gamma(T)
$$

acting on $\mathcal{F}_{\mathrm{b}}(\mathcal{K})$.

We define the quantized scalar field by

$$
\Phi(g):=\int_{\mathbb{R}^{d}}^{\oplus} \phi(g(x)) d x
$$

on $L^{2}\left(\mathbb{R}^{d} ; \mathcal{F}_{\mathrm{b}}(\mathcal{K})\right)$, where $g: x \in \mathbb{R}^{d} \mapsto g(x) \in \mathcal{K}$. If $g$ is a strongly continuous function, then $\Phi(g)$ is self-adjoint (see [12, Theorem XIII.85 (b)]).

Now we define a total Hamiltonian $H$ acting on $\mathcal{F}$ by

$$
H:=-\Delta \otimes I+I \otimes H_{\mathrm{b}}+g H_{\mathrm{I}},
$$

where $g \in \mathbb{R}$ denotes a coupling constant and

$$
H_{\mathrm{I}}:=\sum_{j=1}^{J} B_{j} \otimes \Phi\left(g_{j}\right)
$$

Here, $B_{j}(j=1, \cdots, J)$ are bounded self-adjont operators on $\mathcal{H}$. The functions $g_{j}(j=1, \cdots, J, J \in \mathbb{N})$ from $\mathbb{R}^{d}$ to $\mathcal{K}$ are strongly continuous.

Example 3.1 (the Nelson model) If $\operatorname{dim} \mathcal{H}=1$ and $B_{j}=1$, then the Hamiltonian $H$ is written as

$$
H_{\text {Nelson }}:=-\Delta \otimes I+I \otimes H_{\mathrm{b}}+g \sum_{j=1}^{J} \Phi\left(g_{j}\right)
$$

on $L^{2}\left(\mathbb{R}^{d} ; \mathcal{F}_{\mathrm{b}}(\mathcal{K})\right)$, which is called the Nelson Hamiltonian. The weak coupling limit of this Hamiltonian is studied by Hiroshima $[6,7]$. 


\subsection{Scaling limit for the model}

To begin with, we introduce a scaled Hamiltonian $H(\Lambda)(\Lambda>0)$ by

$$
H(\Lambda):=-\Delta \otimes I+\Lambda^{2} I \otimes H_{\mathrm{b}}+g \Lambda H_{\mathrm{I}}
$$

In order to describe our result, we introduce some notations, and formulate our assumption.

We denote by $L^{\infty}\left(\mathbb{R}^{d} ; \mathcal{K}\right)$ the set of measurable functions $f: \mathbb{R}^{d} \mapsto \mathcal{K}$ for which

$$
\|f\|_{\infty}:=\operatorname{ess} \sup _{x \in \mathbb{R}^{d}}\|f(x)\|_{\mathcal{K}}<\infty .
$$

For $\alpha \in \mathbb{R}$, we define a $\mathcal{K}$-valued function $T^{\alpha} f$ on $\mathbb{R}^{d}$ as follows: if $f(x) \in$ $D\left(T^{\alpha}\right)$ a.e. $x \in \mathbb{R}^{d}$ with respect to Lebesuge mesure,

$$
\left(T^{\alpha} f\right)(x):=T^{\alpha} f(x) .
$$

Definition 3.1 Let $\alpha \in \mathbb{R} . L_{\alpha}^{\infty}\left(\mathbb{R}^{d} ; \mathcal{K}\right)$ denotes the set of $\mathcal{K}$-valued functions $f$ on $\mathbb{R}^{d}$ satisfing the following conditions:

(i) $f$ is strongly continuous with $f \in L^{\infty}\left(\mathbb{R}^{d} ; \mathcal{K}\right)$.

(ii) $f(x) \in D\left(T^{\alpha}\right)(x \in \mathbb{R})$ and $T^{\alpha} f \in L^{\infty}\left(\mathbb{R}^{d} ; \mathcal{K}\right)$.

A $\mathcal{K}$-valued function $f$ on $\mathbb{R}^{d}$ is said to be differentiable with respect to $x_{\mu}$ if the net

$$
\frac{f\left(x_{1}, \cdots, x_{\mu}+\varepsilon, \cdots, x_{d}\right)-f(x)}{\varepsilon}
$$

converges as $\varepsilon \rightarrow 0$ for any $x=\left(x_{1}, \cdots, x_{d}\right) \in \mathbb{R}^{d}$. Then, we denote the limit of (3.8) by $\partial_{\mu} f$. One can define the $n$ times diferentiability $(n \in \mathbb{N})$, inductively:

$$
\partial_{\mu}^{n} f:=\partial_{\mu}\left(\partial_{\mu}^{(n-1)} f\right), n \geq 1 .
$$

Hypothesis I The functions $g_{j}(j=1, \cdots, J)$ are twice diferensiable and satisfy the following conditions:

(i) $g_{j} \in L_{-3 / 2}^{\infty}\left(\mathbb{R}^{d} ; \mathcal{K}\right)$.

(ii) $\partial_{\mu}\left(T^{-1} g_{j}\right) \in L_{-1 / 2}^{\infty}\left(\mathbb{R}^{d} ; \mathcal{K}\right) \cap L_{1 / 2}^{\infty}\left(\mathbb{R}^{d} ; \mathcal{K}\right)$ for $\mu=1, \cdots, d$.

(iii) $\partial_{\mu}^{2}\left(T^{-1} g_{j}\right) \in L_{-1 / 2}^{\infty}\left(\mathbb{R}^{d} ; \mathcal{K}\right)$ for $\mu=1, \cdots, d$.

Moreover, we assume that for any $j, k=1, \cdots, J$ and $x \in \mathbb{R}^{d}$

$$
\left\langle g_{j}(x), g_{k}(x)\right\rangle, \quad\left\langle g_{j}(x), T^{-1} g_{k}(x)\right\rangle, \quad\left\langle T^{-1} g_{j}(x), T^{-1} g_{k}(x)\right\rangle \in \mathbb{R} .
$$


We are now ready to describe our result. Let

$$
V_{\mathrm{eff}}=-\frac{g^{2}}{2} \sum_{1 \leq j, k \leq J} B_{k} B_{j} V_{j, k},
$$

on $L^{2}\left(\mathbb{R}^{d} ; \mathcal{H}\right)$, where

$$
V_{j, k}(x)=\left\langle g_{j}(x), T^{-1} g_{k}(x)\right\rangle, \text { a.e. } x \in \mathbb{R} .
$$

Let $\Omega:=\{1,0,0, \cdots\} \in \mathcal{F}_{\mathrm{b}}(\mathcal{K})$, the Fock vacuum, and $P_{0}$ be the orthogonal projection onto the subspace $\{\alpha \Omega \mid \alpha \in \mathbb{C}\}=\operatorname{ker} H_{\mathrm{b}}$.

Theorem 3.1 Assume Hypothesis I. Let $z \in \mathbb{C} \backslash \mathbb{R}$ or $z<0$ with $|z|$ sufficiently large. Then,

$$
\text { S- } \lim _{\Lambda \rightarrow \infty}(H(\Lambda)-z)^{-1}=\left(H_{\mathrm{eff}}-z\right)^{-1} \otimes P_{0},
$$

where

$$
H_{\mathrm{eff}}=-\Delta+V_{\mathrm{eff}}
$$

on $L^{2}\left(\mathbb{R}^{d} ; \mathcal{H}\right)$.

\section{Proof of Theorem 3.1}

Note that the following facts hold: Let $f_{1} \in L_{-1 / 2}^{\infty}\left(\mathbb{R}^{d} ; \mathcal{K}\right)$. Then, $D\left(H_{\mathrm{b}}^{1 / 2}\right) \subset$ $D\left(\Phi\left(f_{1}\right)\right)$ and for some constans $c\left(f_{1}\right), d\left(f_{1}\right) \geq 0$

$$
\left\|\Phi\left(f_{1}\right) \Psi\right\| \leq c\left(f_{1}\right)\left\|I \otimes H_{\mathrm{b}}^{1 / 2} \Psi\right\|+d\left(f_{1}\right)\|\Psi\|, \quad \Psi \in D\left(I \otimes H_{\mathrm{b}}^{1 / 2}\right) .
$$

If $f_{2} \in L_{-1 / 2}^{\infty}\left(\mathbb{R}^{d} ; \mathcal{K}\right) \cap L_{1 / 2}^{\infty}\left(\mathbb{R}^{d} ; \mathcal{K}\right)$, then $D\left(I \otimes H_{\mathrm{b}}\right) \subset D\left(\Phi\left(f_{1}\right) \Phi\left(f_{2}\right)\right)$ and for some constants $c\left(f_{1}, f_{2}\right), d\left(f_{1}, f_{2}\right) \geq 0$

$$
\left\|\Phi\left(f_{1}\right) \Phi\left(f_{2}\right) \Psi\right\| \leq c\left(f_{1}, f_{2}\right)\left\|I \otimes H_{\mathrm{b}} \Psi\right\|+d\left(f_{1}, f_{2}\right)\|\Psi\|, \quad \Psi \in D\left(I \otimes H_{\mathrm{b}}\right) .
$$

These facts imply that $\Phi\left(f_{1}\right)$ is $I \otimes H_{\mathrm{b}}^{1 / 2}$-bounded and $\Phi\left(f_{1}\right) \Phi\left(f_{2}\right)$ is $I \otimes$ $H_{\mathrm{b}}$-bounded. Hence, using the method in [13], we can prove Theorem 3.1. Throughout this section, it is taken for granted that Hypothesis I hold. 


\subsection{Unitary transformation}

Let

$$
\mathcal{F}_{\text {fin }}(\mathcal{M}):=\mathscr{L}\left\{\Omega, a\left(f_{1}\right)^{*} \cdots a\left(f_{n}\right)^{*} \Omega \mid n \geq 1, f_{j} \in \mathcal{M}, j=1, \cdots, n\right\},
$$

for a subset $\mathcal{M}$ of $\mathcal{K}$. For every $D \subset L^{2}\left(\mathbb{R}^{d}\right)$ and $\mathcal{E} \subset \mathcal{F}_{\mathrm{b}}(\mathcal{K})$, let

$$
D \cdot \mathcal{E}:=\mathscr{L}\{f \times \psi \mid f \in D, \psi \in \mathcal{E}\} \subset L^{2}\left(\mathbb{R}^{d} ; \mathcal{F}_{\mathrm{b}}(\mathcal{K})\right)
$$

where

$$
(f \times \psi)(x):=f(x) \psi \in \mathcal{F}_{\mathrm{b}}(\mathcal{K}), \quad \text { a.e. } x \in \mathbb{R}^{d} .
$$

Now, we introduce a unitary transformation as follows. Let

$$
S=\sum_{j=1}^{J} B_{j} \otimes \Phi\left(i T^{-1} g_{j}\right)
$$

acting on $\mathcal{H} \otimes L^{2}\left(\mathbb{R}^{d} ; \mathcal{F}_{\mathrm{b}}(\mathcal{K})\right)$.

Lemma 4.1 The operator $S$ is essentially self-adjoint.

Proof. Use Nelson's analytic vector theorem. It is easy to see from Hypothesis I (i) that $\mathcal{H} \otimes_{\text {alg }}\left(C_{0}^{\infty}\left(\mathbb{R}^{d}\right) \cdot \mathcal{F}_{\text {fin }}(\mathcal{K})\right)$ is a dense set of analytic vectors of $S$, where where $C_{0}^{\infty}\left(\mathbb{R}^{d}\right)$ denotes the set of infinitely differentiable functions with compact support.

We denote the closure of $S$ by the same symbol $S$. Then, we can define the unitary operator $U(t)(t \in \mathbb{R})$ generated by $S$ :

$$
U(t):=e^{-i t S}, \quad t \in \mathbb{R} .
$$

The subspace

$$
\mathcal{F}_{T}:=\mathcal{H} \otimes_{\text {alg }}\left(C_{0}^{\infty}\left(\mathbb{R}^{d}\right) \cdot \mathcal{F}_{\text {fin }}(D(T)) .\right.
$$

is a core for $-\Delta \otimes I, I \otimes H_{\mathrm{b}}$, and therefore for $-\Delta \otimes I+I \otimes H_{\mathrm{b}}$. It is easy to prove that for $\Psi \in \mathcal{F}_{T}$

$$
\sum_{m, n} \frac{|s|^{n}|t|^{m}}{n ! m !}\left\|S^{n} I \otimes H_{\mathrm{b}} S^{m} \Psi\right\|<\infty, \quad s, t \in \mathbb{R} .
$$

(See [13, Lemma 4.1].) 
Let

$$
E(t)=U(t)\left[\operatorname{ad}^{2}(S) I \otimes H_{\mathrm{b}}\right] U(t)^{-1}, \quad t \in \mathbb{R},
$$

where, for each $X$ in an algebra $\mathcal{A}$, we inductively define $\operatorname{ad}^{N}(X)$ by

$$
\operatorname{ad}^{0}(X) Y=Y, \operatorname{ad}^{N}(X) Y=\left[X, \operatorname{ad}^{N-1}(X) Y\right], \quad Y \in \mathcal{A}, \quad N \geq 1 .
$$

One can prove the following lemma in the same way as in [13, Proposition $4.2]$.

Lemma 4.2 For each $\Psi \in \mathcal{F}_{T}$, there exist $\xi(t), \eta(t) \in[-|t|,|t|]$ such that

$$
U(t) I \otimes H_{\mathrm{b}} U(t)^{-1} \Psi=\left[I \otimes H_{\mathrm{b}}+t H_{\mathrm{I}}-\frac{t^{2}}{2} E(\xi(t))\right] \Psi,
$$

and

$$
U(t) H_{\mathrm{I}} U(t)^{-1} \Psi=\left[H_{\mathrm{I}}-t E(\eta(t))\right] \Psi .
$$

Applying (4.4) and (4.5) with $t=g / \Lambda$, we obtain the following equality:

$$
\begin{aligned}
U(g / \Lambda)\left[\Lambda^{2} I\right. & \left.\otimes H_{\mathrm{b}}+g \Lambda H_{\mathrm{I}}\right] U(g / \Lambda)^{-1} \Psi \\
& =\left[\Lambda^{2} I \otimes H_{\mathrm{b}}-\frac{g^{2}}{2} E(\xi(g / \Lambda))+g^{2} E(\eta(g / \Lambda))\right] \Psi
\end{aligned}
$$

for $\Psi \in \mathcal{F}$.

In what follows, we consider the unitary transformation of $-\Delta \otimes I$ by $U(t)$. Under the condition Hypothesis I, it is easy to show that

$$
\sum_{n, m} \frac{|s|^{n}|t|^{m}}{n ! m !}\left\|S^{n}[-\Delta \otimes I] S^{m} \Psi\right\|<\infty
$$

for any $s, t \in \mathbb{R}$ and $\Psi \in \mathcal{F}_{T}$. Thus, in particular, we can prove

$$
U(t) \mathcal{F}_{T} \subset D(-\Delta \otimes I) \quad(t \in \mathbb{R}) .
$$

We set

$$
\delta A(t):=U(t)[-\Delta \otimes I] U(t)^{-1}-[-\Delta \otimes I],
$$

Then, we can write

$$
\begin{aligned}
& U(g / \Lambda)^{-1} H(\Lambda) U(g / \Lambda) \Psi \\
& \quad=\left[-\Delta \otimes I+\Lambda^{2} I H_{\mathrm{b}}+C_{\Lambda}\right] \Psi
\end{aligned}
$$


for $\Psi \in \mathcal{F}_{T}$, where we have set

$$
C_{\Lambda}:=\delta A(g / \Lambda)-\frac{g^{2}}{2} E(\xi(g / \Lambda))+g^{2} E(\eta(g / \Lambda)) .
$$

We denote the closure of $C_{\Lambda}$ by the same symbol. Indeed, we can prove the following theorem:

Theorem 4.3 Let

$$
\widehat{H}(\Lambda):=-\Delta \otimes I+\Lambda^{2} I \otimes H_{\mathrm{b}}+C_{\Lambda} .
$$

Then, $\widehat{H}(\Lambda)$ is essentilly self-adjoint on $\mathcal{F}_{T}$ and the following operator equality holds:

$$
U(g / \Lambda) H(\Lambda) U(g / \Lambda)^{-1}=\widehat{H}(\Lambda)
$$

We prove Theorem 4.3 in the following subsection.

\subsection{Uniform relative boundedness of $C_{\Lambda}$}

Let

$$
H_{0}(\Lambda):=-\Delta \otimes I+\Lambda^{2} I \otimes H_{\mathrm{b}} .
$$

In this section, we prove that $C_{\Lambda}$ is $H_{0}(\Lambda)$-infinitesimally small uniformly near $\infty$. As a result, we obtain Theorem 4.3.

To begin with, we state a uniform relative boundedness of the operator $E(t)$ :

Lemma 4.4 The operator $E(t)$ defined by (4.3) is $I \otimes H_{\mathrm{b}}$-bounded uniformly near 0 .

Proof. It is easy to see that

$$
\begin{aligned}
\| E(\xi(t) \Psi \| \leq & \left(\sum_{j, k}\left\|B_{j} B_{k}\right\|\left\|T^{-1} g_{j}\right\|_{\infty}\left\|g_{k}\right\|_{\infty}\right)\|\Psi\| \\
& +\sum_{j, k}\left\|\left[B_{j}, B_{k}\right]\right\|\left\|I \otimes \Phi\left(g_{k}\right) \Phi\left(i T^{-1} g_{j}\right) U(\xi(t))^{-1} \Psi\right\|
\end{aligned}
$$

for all $\Psi \in \mathcal{F}_{T}$. Hence, we need only to prove that $I \otimes \Phi\left(g_{k}\right) \Phi\left(i T^{-1} g_{j}\right) U(\xi(t))^{-1}$ is $I \otimes H_{\mathrm{b}}$-bounded uniformly near 0 . 
For each $\Psi \in \mathcal{F}_{T}$ and $t \in \mathbb{R}$, there exists a constant $\delta(t) \in[-|t|,|t|]$ such that

$$
\begin{aligned}
& U(t) I \otimes \Phi\left(g_{k}\right) \Phi\left(i T^{-1} g_{j}\right) U(t)^{-1} \Psi \\
& =I \otimes \Phi\left(g_{k}\right) \Phi\left(i T^{-1} g_{j}\right) \Psi \\
& \quad-i t U(\delta(t)) \operatorname{ad}^{1}(S)\left[I \otimes \Phi\left(g_{k}\right) \Phi\left(i T^{-1} g_{j}\right)\right] U(\delta(t))^{-1} \Psi
\end{aligned}
$$

(See [13, Lemma 4.5].) By (4.2), the first term on the right hand side in (4.13) is $H_{\mathrm{b}}$-bounded, since $\mathcal{F}_{T}$ is core for $I \otimes H_{\mathrm{b}}$. Therefore, we need only to estimate the second term.

It is easy to see, from (3.9), that

$$
\begin{aligned}
\operatorname{ad}^{1}(S)[I \otimes & \left.\Phi\left(g_{k}\right) \Phi\left(i T^{-1} g_{j}\right)\right] \Psi \\
& =-i \sum_{l} B_{l} \otimes\left\langle T^{-1} g_{l}, g_{k}\right\rangle \Phi\left(i T^{-1} g_{j}\right) \Psi .
\end{aligned}
$$

Hence, we obtain the following:

$$
\begin{aligned}
& \left\|I \otimes \Phi\left(g_{k}\right) \Phi\left(i T^{-1} g_{j}\right) U(t)^{-1} \Psi\right\| \\
& \quad=\left\|I \otimes \Phi\left(g_{k}\right) \Phi\left(i T^{-1} g_{j}\right) \Psi\right\| \\
& \quad+|t| \sum_{l}\left\|B_{l}\right\|\left\|T^{-1} g_{l}\right\|_{\infty}\left\|g_{k}\right\|_{\infty}\left\|I \otimes \Phi\left(i T^{-1} g_{j}\right) \Psi\right\|,
\end{aligned}
$$

where we have used the commutativity of $I \otimes \Phi\left(i T^{-1} g_{j}\right)$ and $U(\delta(t))^{-1}$. One can assume that $|t| \leq 1$ without loss of generality. Note that $|\xi(\delta(t))| \leq|t|$. Thus, by (4.1) and (4.2), $I \otimes \Phi\left(g_{k}\right) \Phi\left(i T^{-1} g_{j}\right) U(\xi(\delta(t)))^{-1} \Psi$ is $I \otimes H_{\mathrm{b}}$-bounded uniformly near 0 . Hence, we obtain the desired result.

By (4.9), we need only to prove the uniform relative boundedness of the operator $\delta A(t)$. The following lemma is a fundamental fact to do this.

Lemma 4.5 The operator $U(t) I \otimes H_{\mathrm{b}} U(t)^{-1}$ is $I \otimes H_{\mathrm{b}}$-bounded uniformly near 0 .

Proof. By Lemma 4.2,

$$
\left\|U(t) I \otimes H_{\mathrm{b}} U(t)^{-1} \Psi\right\| \leq\left\|I \otimes H_{\mathrm{b}} \Psi\right\|+|t|\left\|H_{\mathrm{I}} \Psi\right\|+\frac{|t|^{2}}{2}\|E(\xi(t)) \Psi\|
$$

for all $\Psi \in \mathcal{F}_{T}$. From Lemma 4.4, $E(\xi(t))$ is $I \otimes H_{\mathrm{b}}$-bounded unifomly near 0 . Therefore, we need only to prove that $H_{\mathrm{I}}$ is $\left(I \otimes H_{\mathrm{b}}\right)$-bounded unifomly near 0 , since we can assume that $|t| \leq 1$ without loss of generality. 
It is easy to see that

$$
\left\|H_{I} \Psi\right\| \leq \sum_{j=1}^{J}\left\|B_{j}\right\|\left\|I \otimes \Phi\left(g_{j}\right) \Psi\right\|
$$

for all $\Psi \in \mathcal{F}_{T}$. Hence, we obtain the desired result by (4.1).

The following lemma follows from (4.1), (4.2) and Lemma 4.5.

Lemma 4.6 Let $f_{1} \in L_{-1 / 2}^{\infty}\left(\mathbb{R}^{d} ; \mathcal{K}\right)$ and $f_{2} \in L_{-1 / 2}^{\infty}\left(\mathbb{R}^{d} ; \mathcal{K}\right) \cap L_{1 / 2}^{\infty}\left(\mathbb{R}^{d} ; \mathcal{K}\right)$. Then, the operator $U(t)\left[I \otimes \Phi\left(f_{1}\right)\right] U(t)^{-1}$ is $I \otimes H_{\mathrm{b}}$-infinitesimally small unifomly near 0 and $U(t)\left[I \otimes \Phi\left(f_{1}\right) \Phi\left(f_{2}\right)\right] U(t)^{-1}$ is $I \otimes H_{\mathrm{b}}$-bounded uniformly near 0 .

In what follows, we describe a uniform relative boundedness of the operator $\delta A(t)$.

Lemma 4.7 The operator $\delta A(t)$ is $-\Delta \otimes I+I \otimes H_{\mathrm{b}}$-infinitesimally small uniformly near 0 and for all $\Psi \in \mathcal{F}_{T}$

$$
\text { s- } \lim _{t \rightarrow \infty} \delta A(t) \Psi=0
$$

Proof. It is easy to see that $U(t) \Psi \in D(\Delta \otimes I)$ for all $t \in \mathbb{R}$ and that

$$
\delta A(t) \Psi=-\left[U(t)(\Delta \otimes I) U(t)^{-1}-\Delta \otimes I\right] \Psi
$$

for all $\Psi \in \mathcal{F}_{T}$. In the same way as in Lemma 4.2, one can prove that there exists $\omega(t) \in[-|t|,|t|](t \in \mathbb{R})$ such that

$$
\left[U(t)(\Delta \otimes I) U(t)^{-1}-\Delta \otimes I\right] \Psi=-i t U(\omega(t)) \operatorname{ad}^{1}(S)[\Delta \otimes I] U(\omega(t))^{-1} \Psi .
$$

Thus, under the identification (3.4), we have

$$
\|\delta A(t) \Psi\| \leq|t| \sum_{j=1}^{J}\left\|B_{j}\right\|\left\|I \otimes\left[\Phi\left(i T^{-1} g_{j}\right), \Delta\right] U(\omega(t))^{-1} \Psi\right\|
$$

for all $\Psi \in \mathcal{F}_{T}$. This inequality implies that (4.14) holds.

We need only to prove a uniform relative boundedness of the operator

$$
I \otimes\left[\Phi\left(i T^{-1} g_{j}\right), \Delta\right] U(\omega(t))^{-1} .
$$


Let $D_{\mu}(\mu=1, \cdots, d)$ be the partial differential operators on $L^{2}\left(\mathbb{R}^{d} ; \mathcal{F}_{\mathrm{b}}(\mathcal{K})\right)$. It is is easy to see that

$$
\left[\Phi\left(i T^{-1} g_{j}\right), \Delta\right]=\sum_{\mu=1}^{d}\left(D_{\mu}\left[\Phi\left(i T^{-1} g_{j}\right), D_{\mu}\right]+\left[\Phi\left(i T^{-1} g_{j}\right), D_{\mu}\right] D_{\mu}\right)
$$

on $C_{0}^{\infty}\left(\mathbb{R}^{d}\right) \cdot \mathcal{F}_{\text {fin }}(\mathcal{K})$. One can easily prove that

$$
\left[\Phi\left(i T^{-1} g_{j}\right), D_{\mu}\right] \psi=-\Phi\left(i \partial_{\mu} T^{-1} g_{j}\right) \psi
$$

for all $\psi \in C_{0}^{\infty}\left(\mathbb{R}^{d}\right) \cdot \mathcal{F}_{\text {fin }}(\mathcal{K})$. Hence, we obtain the following:

$$
\begin{aligned}
{\left[\Phi\left(i T^{-1} g_{j}\right), \Delta\right] \psi } & =-\sum_{\mu=1}^{d}\left(D_{\mu} \Phi\left(i \partial_{\mu} T^{-1} g_{j}\right)+\Phi\left(i \partial_{\mu} T^{-1} g_{j}\right) D_{\mu}\right) \psi \\
& =-\sum_{\mu=1}^{d}\left(\Phi\left(i \partial_{\mu}^{2} T^{-1} g_{j}\right)+2 \Phi\left(i \partial_{\mu} T^{-1} g_{j}\right) D_{\mu}\right) \psi
\end{aligned}
$$

Thus, we get the following inequality.

$$
\left\|\left[\Phi\left(i T^{-1} g_{j}\right), \Delta\right] \psi\right\| \leq \sum_{\mu=1}^{d}\left\|\left[\Phi\left(i \partial_{\mu}^{2} T^{-1} g_{j}\right)+2 \Phi\left(i \partial_{\mu} T^{-1} g_{j}\right) D_{\mu}\right] \psi\right\|
$$

for all $\psi \in C_{0}^{\infty}\left(\mathbb{R}^{d}\right) \cdot \mathcal{F}_{\text {fin }}(\mathcal{K})$. By $(4.15)$, we get

$$
\begin{aligned}
\|\delta A(t) \Psi\| \leq|t| & \sum_{j=1}^{J}\left\|B_{j}\right\|\left\|I \otimes \Phi\left(i \partial_{\mu}^{2} T^{-1} g_{j}\right) U(\omega(t))^{-1} \Psi\right\| \\
& +2|t| \sum_{j=1}^{J}\left\|B_{j}\right\|\left\|I \otimes \Phi\left(i \partial_{\mu} T^{-1} g_{j}\right) D_{\mu} U(\omega(t))^{-1} \Psi\right\|
\end{aligned}
$$

for all $\Psi \in \mathcal{F}_{T}$. By (4.6), the first term on the right hand side is $I \otimes H_{\mathrm{b}}$ infinitesimally small uniformly near 0 . Hence we need only to prove that the operator $I \otimes \Phi\left(i \partial_{\mu} T^{-1} g_{j}\right) D_{\mu} U(\omega(t))^{-1}$ is $-\Delta \otimes I+I \otimes H_{\mathrm{b}}$-bounded uniformly near 0.

Using the same way as in (4.2), one can show that

$$
\begin{aligned}
& U(t) I \otimes \Phi\left(i \partial_{\mu} T^{-1} g_{j}\right) D_{\mu} U(t)^{-1} \Psi \\
& \quad=I \otimes \Phi\left(i \partial_{\mu} T^{-1} g_{j}\right) D_{\mu} \Psi+i t U(\theta(t)) \operatorname{ad}^{1}(S) I \otimes \Phi\left(i \partial_{\mu} T^{-1} g_{j}\right) D_{\mu} U(\theta(t))^{-1} \Psi
\end{aligned}
$$


for all $\Psi \in \mathcal{F}_{T}$ with some $\theta(t) \in[-|t|,|t|]$. Thus, we obtain the following:

$$
\begin{aligned}
\| I & \otimes \Phi\left(i \partial_{\mu} T^{-1} g_{j}\right) D_{\mu} U(t)^{-1} \Psi \| \\
& \leq\left\|I \otimes \Phi\left(i \partial_{\mu} T^{-1} g_{j}\right) D_{\mu} \Psi\right\|+|t|\left\|\operatorname{ad}^{1}(S)\left[I \otimes \Phi\left(i \partial_{\mu} T^{-1} g_{j}\right) D_{\mu}\right] U(\theta(t))^{-1} \Psi\right\| .
\end{aligned}
$$

The first term on the right hand side above is estimated as follows:

$$
\left\|I \otimes \Phi\left(i \partial_{\mu} T^{-1} g_{j}\right) D_{\mu} \Psi\right\| \leq c\left\|I \otimes H_{\mathrm{b}}^{1 / 2} D_{\mu} \Psi\right\|+d\left\|I \otimes D_{\mu} \Psi\right\|
$$

Note that $H_{\mathrm{b}}^{1 / 2} D_{\mu}$ is $-\Delta+H_{\mathrm{b}}$-bounded. We can now estimate the second term on the right hand side of (4.17). It is easy to see that

$$
\begin{aligned}
& \operatorname{ad}^{1}(S)\left[I \otimes \Phi\left(i \partial_{\mu} T^{-1} g_{j}\right) D_{\mu}\right] \\
& =\sum_{l} B_{l} \otimes\left[\Phi\left(i T^{-1} g_{l}\right), \Phi\left(i \partial_{\mu} T^{-1} g_{j}\right) D_{\mu}\right] \\
& =-\sum_{l} B_{l} \otimes \Phi\left(i \partial_{\mu} T^{-1} g_{l}\right) \Phi\left(i \partial_{\mu} T^{-1} g_{j}\right) \\
& \quad+\sum_{l} B_{l} \otimes i \Im\left\langle T^{-1} g_{l}(\cdot), \partial_{\mu} T^{-1} g_{j}(\cdot)\right\rangle D_{\mu} .
\end{aligned}
$$

Thus, one can prove that $U(t) \Psi(t \in \mathbb{R})$ belongs to $D\left(\operatorname{ad}^{1}(S)\left[I \otimes \Phi\left(i \partial_{\mu} T^{-1} g_{j}\right) D_{\mu}\right]\right)$ for all $\Psi \in \mathcal{F}$ and that the following inequality holds:

$$
\begin{aligned}
\left\|\operatorname{ad}^{1}(S)\left[I \otimes \Phi\left(i \partial_{\mu} T^{-1} g_{j}\right) D_{\mu}\right] U(\theta(t))^{-1} \Psi\right\| \\
\leq \sum_{l}\left\|B_{l}\right\|\left\|I \otimes \Phi\left(i \partial_{\mu} T^{-1} g_{l}\right) \Phi\left(i \partial_{\mu} T^{-1} g_{j}\right) U(\theta(t))^{-1} \Psi\right\| \\
\quad+\sum_{l}\left\|B_{l}\right\|\left\|T^{-1} g_{l}\right\|_{\infty}\left\|\partial_{\mu} T^{-1} g_{j}\right\|_{\infty}\left\|I \otimes D_{\mu} U(\theta(t))^{-1} \Psi\right\| .
\end{aligned}
$$

Note that the operator $U(\theta(t)) I \otimes \Phi\left(i \partial_{\mu} T^{-1} g_{l}\right) \Phi\left(i \partial_{\mu} T^{-1} g_{j}\right) U(\theta(t))^{-1}$ is $I \otimes$ $H_{\mathrm{b}}$-bounded uniformly near 0 by Hypothesis I (ii) and Lemma 4.6. Hence, we need only to estimate the second term. Indeed, one can prove that the operator $U(t)\left[I \otimes D_{\mu}\right] U(t)^{-1}-I \otimes D_{\mu}$ is $I \otimes H_{\mathrm{b}}$-infinitesimally small uniformly near 0 , in the same way as in Lemma 4.2. Hence we obtain the desired result.

We can now state a uniform relative boundedness of the operator $C_{\Lambda}$ defined by (4.9). Note that Lemma 2.1 holds. Hence, Lemmas 4.4 and 4.7 imply the following proposition, so that Theorem 4.3 is proved by the KatoRellich theorem.

Proposition 4.8 The operator $C_{\Lambda}$ is $H_{0}(\Lambda)$-infinitesimally small uniformly near $\infty$. 


\subsection{Proof of Theorem 3.1}

We are now ready to prove Theorem 3.1. We need the following proposition:

Proposition 4.9 For all $\Psi \in \mathcal{F}_{T}$

$$
\text { s- } \lim _{\Lambda \rightarrow \infty} C_{\Lambda} \Psi=C \Psi
$$

holds, where

$$
C=\frac{g^{2}}{2} \operatorname{ad}^{2}(S) I \otimes H_{\mathrm{b}}
$$

Proof. By (4.14), we need only to prove

$$
\text { s- } \lim _{t \rightarrow 0} E(t) \Psi=E \Psi
$$

where

$$
E=\operatorname{ad}^{2}(S) I \otimes H_{\mathrm{b}} .
$$

Note that the following fact holds:

$$
E(t) \Psi=\left\{E-i t U(\kappa(t))\left[\operatorname{ad}^{1}(S) E\right] U(\kappa(t))^{-1}\right\} \Psi
$$

with some $\kappa(t) \in[-|t|,|t|](t \in \mathbb{R})$. Therefore, we obtain the desired result.

Now, we apply Corollary 2.4 to $H(\Lambda)$. Let

$$
C_{\infty}:=\left(I \otimes P_{0}\right) C\left(I \otimes P_{0}\right),
$$

where we have denoted the closure of the operator $C$ by the same notation. Note that

$$
(\widehat{H}(\Lambda)-z)^{-1}=U(g / \Lambda)(H(\Lambda)-z)^{-1} U(g / \Lambda)^{-1}
$$

for all $z$ contained in the resolvent set of $H(\Lambda)$. It is easy to see that

$$
\text { s- } \lim _{t \rightarrow \infty} U(t)=I \text {. }
$$

By Proposition 4.8 and 4.9, we obtain the following: For $z \in \mathbb{C} \backslash \mathbb{R}$

$$
\text { S- } \lim _{\Lambda \rightarrow \infty}(H(\Lambda)-z)^{-1}=\left(-\Delta \otimes I+C_{\infty}\right)^{-1} I \otimes P_{0}
$$

holds. 
By [1, Theorem 2.12] we need only to prove that the partial expectaion of $C$ with respect to the Fock vacuum $\Omega$ is eaqual to $V_{\text {eff }}$ defined by (3.10). It is easy to see that

$$
\begin{aligned}
\left\langle u_{1} \otimes f_{1}\right. & \left.\otimes \Omega, C\left(u_{2} \otimes f_{2} \otimes \Omega\right)\right\rangle \\
& =-\frac{g^{2}}{2} \sum_{j, k}\left\langle f_{1}, V_{j, k} f_{2}\right\rangle\left\langle u_{1},\left(\frac{1}{2}\left[B_{j}, B_{k}\right]+B_{k} B_{j}\right) u_{2}\right\rangle
\end{aligned}
$$

for all $u_{i} \in \mathcal{H}, f_{i} \in L^{2}\left(\mathbb{R}^{d}\right)(i=1,2)$. Note that $\left[B_{j}, B_{k}\right]=-\left[B_{k}, B_{j}\right]$. It follows that

$$
\left\langle u_{1} \otimes f_{1} \otimes \Omega, C\left(u_{2} \otimes f_{2} \otimes \Omega\right)\right\rangle=\left\langle u_{1} \otimes f_{1} \otimes \Omega, V_{\text {eff }} u_{2} \otimes f_{2} \otimes \Omega\right\rangle .
$$

Hence, we obtain that

$$
C_{\infty}=V_{\text {eff }} \otimes P_{0}
$$

and therefore (3.12) holds.

\section{Nucleon-Pion interaction}

In this section, we give a concrete realization of the abstract model $H$, which is an interaction model between nucleons and pions with isospin (see [13, Section 5.1]).

Let $\sigma_{j}, \tau_{j}(j=1,2,3)$ be the Pauli matrices:

$$
\sigma_{1}=\tau_{1}=\left(\begin{array}{ll}
0 & 1 \\
1 & 0
\end{array}\right), \quad \sigma_{2}=\tau_{2}=\left(\begin{array}{cc}
0 & -i \\
i & 0
\end{array}\right), \quad \sigma_{3}=\tau_{3}=\left(\begin{array}{cc}
1 & 0 \\
0 & -1
\end{array}\right),
$$

and

$$
\begin{gathered}
\sigma_{j}{ }^{(i)}=1_{2} \otimes \cdots \otimes \widetilde{\sigma}_{j}^{i \text { th }} \otimes \cdots \otimes 1_{2}, \quad j=1,2,3, \\
\tau_{\alpha}{ }^{(i)}=1_{2} \otimes \cdots \otimes{\stackrel{\text { th }}{\tau_{\alpha}}} \otimes \cdots \otimes 1_{2}, \quad \alpha=1,2,3,
\end{gathered}
$$

where $1_{2}$ is the $2 \times 2$ identity matrix. Physically, $\sigma^{(i)}=\left(\sigma_{1}{ }^{(i)}, \sigma_{2}{ }^{(i)}, \sigma_{3}{ }^{(i)}\right)$ and $\tau^{(i)}=\left(\tau_{1}{ }^{(i)}, \tau_{2}{ }^{(i)}, \tau_{3}{ }^{(i)}\right)$ denote the spin and the isospin of the $i$ th particle, respectively. Set

$$
\mathcal{H}_{N}:=\left[\bigotimes^{N} \mathbb{C}^{2}\right] \bigotimes\left[\bigotimes^{N} \mathbb{C}^{2}\right]
$$

If there is no danger of confusion, we denote the operators $\sigma_{j}^{(i)} \otimes\left(\otimes^{N} 1_{2}\right)$ and $\left(\otimes^{N} 1_{2}\right) \otimes \tau_{\alpha}{ }^{(i)}$ acting on $\mathcal{H}_{N}$ by the same symbol $\sigma_{j}{ }^{(i)}$ and $\tau_{\alpha}{ }^{(i)}$, respectively. We denote by $\hbar$ the Planck constant divided by $2 \pi$. Put

$$
B_{j, \alpha}^{(i)}:=\frac{\hbar}{2} \sigma_{j}{ }^{(i)} \tau_{\alpha}{ }^{(i)}, \quad i=1, \cdots, N, j, \alpha=1,2,3,
$$


which act on $\mathcal{H}_{N}$. It is straightforward to see that

$$
\left[B_{j, \alpha}^{(i)}, B_{k, \beta}^{(l)}\right]=0, \quad i \neq l, j, k, \alpha, \beta=1,2,3 .
$$

By the anticommutativity of the Pauli matrices, it follows that, for $i=$ $1, \cdots, N, j, k, \alpha, \beta=1,2,3$,

$$
\left\{B_{j, \alpha}^{(i)}, B_{k, \beta}^{(i)}\right\}=\frac{\hbar^{2}}{4} \delta_{j k} \delta_{\alpha \beta},
$$

where $\{X, Y\}=X Y+Y X$ and $\delta_{i j}$ is Kronecker's delta.

We denote by $m$ and $c$ the mass of a pion and the speed of light, respectively. Let

$$
\omega(k)=\sqrt{\hbar^{2} k^{2} c^{2}+m^{2} c^{4}}\left(k \in \mathbb{R}^{3}\right),
$$

where $\omega$ denotes a dispersion relation of one free pion. Let

$$
\mathcal{K}=\bigoplus^{3} L^{2}\left(\mathbb{R}^{3}\right)
$$

The function $\omega$ defines a multiplication operator on $\mathcal{K}$. We denote it by the same symbol $\omega$ :

$$
\omega f:=\left(\omega f_{1}, \omega f_{2}, \omega f_{3}\right), \quad f=\left(f_{1}, f_{2}, f_{3}\right) \in \mathcal{K}
$$

with $f_{i} \in D(\omega)$. Let $H_{\mathrm{b}}=d \Gamma(\omega)$. Then, $H_{\mathrm{b}}$ represents the free Hamiltonian of the pion field.

Let

$$
\phi_{\alpha}(f):=\phi\left(f_{\alpha}\right), \quad f \in L^{2}\left(\mathbb{R}^{3}\right),
$$

where

$$
f_{\alpha}:=\left(\delta_{\alpha 1} f, \delta_{\alpha 2} f, \delta_{\alpha 3} f\right) .
$$

We denote by $\rho$ the density of a nucleon, which is a real distribution satisfying $\widehat{\partial_{j}} \rho / \sqrt{\omega} \in L^{2}\left(\mathbb{R}^{3}\right)$, where $\widehat{\partial_{j} \rho}$ denotes the Fourier transform of $\partial_{j} \rho$. Let

$$
\Phi_{\alpha}\left(g_{j}^{(i)}\right)=\int_{\mathbb{R}^{3 N}}^{\oplus} \phi_{\alpha}\left(g_{j}^{(i)}(x)\right) d x
$$

where

$$
g_{j}^{(i)}(x)=-\frac{\sqrt{\hbar}}{\sqrt{(2 \pi)^{3} \omega}} \widehat{\hbar \partial_{j} \rho} e^{-i k \cdot x_{i}}
$$

for $x:=\left(x_{1}, \cdots, x_{N}\right) \in \mathbb{R}^{3 N}$. Here $x_{i} \in \mathbb{R}^{3}$ indicates the coordinate of the $i$ th nucleon. 
A Hamiltonian of spin-nucleons interacting with pions, acting on $\mathcal{H}_{N} \otimes$ $L^{2}\left(\mathbb{R}^{3 N} ; \mathcal{F}_{\mathrm{b}}(\mathcal{K})\right)$, is defined by

$H(\hbar, c, M):=-\frac{\hbar^{2}}{2 M} \Delta \otimes I+\frac{\hbar}{2} \sum_{i=1}^{N} \sigma_{3}{ }^{(i)} \otimes I+I \otimes H_{\mathrm{b}}+g \sum_{\substack{1 \leq i \leq N \\ 1 \leq j, \alpha \leq 3}} B_{j, \alpha}^{(i)} \otimes \Phi_{\alpha}\left(g_{j}{ }^{(i)}\right)$,

where $g \in \mathbb{R}$ is a coupling constant.

Now, we define the scaled Hamiltonian by

$$
H(\Lambda):=\frac{1}{\Lambda^{2}} H\left(\Lambda^{2} \hbar, \Lambda^{2} c, \Lambda^{2} M\right) .
$$

Then, we can write

$$
H(\Lambda)=-\frac{\hbar^{2}}{2 M} \Delta \otimes I+\frac{\hbar}{2} \sum_{i=1}^{N} \sigma_{3}{ }^{(i)} \otimes I+\Lambda^{2} I \otimes H_{\mathrm{b}}+g \Lambda H_{\mathrm{I}},
$$

where

$$
H_{\mathrm{I}}=\sum_{i=1}^{N} \sum_{1 \leq j, \alpha \leq 3} B_{j, \alpha}^{(i)} \otimes \Phi_{\alpha}\left(g_{j}^{(i)}\right)
$$

We now ready to derive a quantum mechanical Hamiltonian from $H(\Lambda)$. Let

$$
H_{\mathrm{eff}}=-\frac{\hbar^{2}}{2 M} \Delta+\frac{\hbar}{2} \sum_{i=1}^{N} \sigma_{3}^{(i)}+\sum_{1 \leq i<l \leq N} E_{i, l}+N E_{0}
$$

where

$$
E_{i, l}(x)=-\frac{g^{2} \hbar}{(2 \pi)^{3}} \int_{\mathbb{R}^{3}}\left(\frac{\hbar}{2} \sigma^{(i)} \cdot \hbar k\right)\left(\frac{\hbar}{2} \sigma^{(l)} \cdot \hbar k\right) \frac{|\hat{\rho}(k)|^{2}}{\omega(k)^{2}} e^{-i k \cdot\left(x_{i}-x_{l}\right)} d k,
$$

a.e. $x=\left(x_{1}, \cdots, x_{N}\right) \in \mathbb{R}^{3 N}$ and

$$
E_{0}=-\frac{3}{2} \frac{g^{2} \hbar}{(2 \pi)^{3}} \sum_{\alpha=1}^{3} \tau_{\alpha}^{(i)} \tau_{\alpha}{ }^{(l)}\left(\frac{\hbar}{2}\right)^{2} \int_{\mathbb{R}^{3}}|\hbar k|^{2} \frac{|\hat{\rho}(k)|^{2}}{\omega(k)^{2}} d k
$$

Theorem 5.1 Suppose that

$$
\omega^{-3 / 2} g_{j}{ }^{(i)} \in L^{2}\left(\mathbb{R}^{3}\right), \quad i=1, \cdots, N, \quad j=1,2,3 .
$$

Then, for all $z \in \mathbb{C} \backslash \mathbb{R}$ or $z<0$ with sufficiently large,

$$
\text { S- } \lim _{\Lambda \rightarrow \infty}(H(\Lambda)-z)^{-1}=\left(H_{\text {eff }}-z\right)^{-1} \otimes P_{0} .
$$


Proof. Applying Theorem 3.1, we have

$$
\text { S- } \lim _{\Lambda \rightarrow \infty}(H(\Lambda)-R \otimes I-z)^{-1}=\left(H_{\mathrm{eff}}-R-z\right)^{-1} \otimes P_{0},
$$

where

$$
R=\frac{\hbar}{2} \sum_{i=1}^{N} \sigma_{3}{ }^{(i)} .
$$

Since $R$ is bounded, we can prove the desired result in the same way as in Theorem 2.3.

Remark 5.1 Physically, $E_{0}$ and $E_{i, l}$ above are considered respectively as the self-energy of each nucleon and an effective potential of the force between two nucleons caused by the exchange of pions.

Acknowledgments The author would like to thank Professor A. Arai for useful comments.

\section{References}

[1] A. Arai, An asymptotic analysis and its application to the nonrelativistic limit of the Pauli-Fierz and a spin-boson model, J. Math. Phys. 31 (1990), 2653-2663.

[2] A. Arai, Fock Spaces and Quantum Fields, Nippon-Hyouronsya, Tokyo, 2000 (in Japanese)

[3] A. Arai and M. Hirokawa, On the existence and uniquness of ground states of a generalized spin-boson model, J. Funct. Anal. 151 (1997), 455-503.

[4] A. Arai and H. Kawano, Enhanced binding in a general class of quantum field models, Rev. Math. Phys. 15 (2003), 387-423.

[5] E. B. Davies, Particle-boson interactions and the weak coupling limit, J. Math. Phys. 20 (1979), 345-351.

[6] F. Hiroshima, Weak coupling limit with a removal of an ultraviolet cutoff for a Hamiltonian of particles interacting with a massive scalar field, Inf. Dimen. Anal. Quantum Prob. Relat. Top. 1 (1998), 407-423. 
[7] F. Hiroshima, Weak coupling limit and removing an ultraviolet cutoff for a Hamiltonian of particles interacting with a quantized scalar field, J. Math. Phys. 40 (1999), 1215-1236.

[8] F. Hiroshima, Observable effects and parametrized scaling limits of a model in nonrelativistic quantum electrodynamics, J. Math. Phys. 43 (2002), 1755-1795.

[9] E. Nelson, Interaction of nonrelativistic particles with a quantized scalar field, J. Math. Phys. 5 (1964), 1190-1197.

[10] M. Reed and B. Simon, Methods of Modern Mathmatical Physics Vol. I, Academic Press, New York (1972).

[11] M. Reed and B. Simon, Methods of Modern Mathmatical Physics Vol. II, Academic Press, New York (1975).

[12] M. Reed and B. Simon, Methods of Modern Mathmatical Physics Vol. IV, Academic Press, New York (1978).

[13] A. Suzuki, A scaling limit for a general class of quantum field models and its application to nuclear physics and condenced matter physics, preprint (2005). 